\title{
Subliminal faces with different valence: Unconscious mismatch detection indicates interactions between unconscious processing
}

\author{
Shen Tu${ }^{1,2,3^{*}}$, Ulla Martens ${ }^{4 *}$, Guang Zhao ${ }^{5}$, Weigang Pan ${ }^{1,2}$, Ting Wang ${ }^{1,2}$, Jiang Qiu ${ }^{1,2}$, \\ Qinglin Zhang ${ }^{1,2}$ \\ ${ }^{1}$ School of Psychology, Southwest University, Chongqing, China \\ ${ }^{2}$ Key Laboratory of Cognition and Personality, Ministry of Education, Chongqing, China \\ ${ }^{3}$ Psychology, Institute of Education, China West Normal University, Nanchong, China \\ ${ }^{4}$ Institute of Experimental Psychology, University of Osnabrück, Osnabrück, Germany \\ ${ }^{5}$ School of Psychology, Liaoning Normal University, Dalian, China \\ Email: shentu614@yeah.net, zhangq1@swu.edu.cn
}

Received 25 September 2013; revised 28 October 2013; accepted 4 November 2013

Copyright (C) 2013 Shen Tu et al. This is an open access article distributed under the Creative Commons Attribution License, which permits unrestricted use, distribution, and reproduction in any medium, provided the original work is properly cited.

\begin{abstract}
Many studies revealed unconscious effects on conscious processing. However, in this study, we tried to investigate whether unconscious processes could interact with each other by using simultaneously presented face pictures with the same or a different unconscious valence (SUV versus DUV). In the first event-related potential (ERP) study, DUV elicited a smaller N2 as compared with SUV. In the second functional magnetic resonance imaging (fMRI) experiment, the left middle frontal gyrus (MFG) was activated under DUV condition in comparison to SUV condition. These results support the idea of interactions between unconscious processes (unconscious mismatch detection). The theoretical implications are discussed in the light of the global neuronal workspace theory.
\end{abstract}

Keywords: Interactions between Unconscious Processing; Unconscious Mismatch Detection; Global Neuronal Workspace Theory; fMRI; ERP

\section{INTRODUCTION}

Many studies have shown that unconscious information processes could affect subsequent processing of different information [1-5]. In the meantime, other studies have demonstrated that consciousness modulates unconscious processes [6-11]. The question arises whether interactions between unconscious processes can occur.

*The authors contributed equally to this work.
One indication for a possible unconscious influence on unconscious processing was found in two studies $[12,13]$. Here, the unconscious stimulus (erotic pictures and impending threat) elicited unconscious spatial attention resulting in behavioral response changes. Obviously, the processing of the unconscious stimulus and the triggering of spatial attention toward a specific unconscious stimulus are different processes. Therefore, these results might demonstrate that unconscious processes influence other unconscious processes. In addition, unconscious processes were generally thought to only involve posterior brain areas as hypothesized in the global neuronal workspace theory $[14,15]$. From a theoretical point, fMRI studies demonstrating that unconscious processes reach prefrontal areas $[16,17]$ are at conflict with the global neuronal workspace theory and suggest that unconscious processes could reach a high level of information processing [17].

In order to investigate the possibility of interactions between unconscious processes, we rely on evidence that subliminally presented facial expressions are processed differently in dependence of their valence [18-24]. In particular, we assume that, in comparison to two subliminal facial expressions of same valence, two subliminal facial expressions of different valence would elicit unconscious mismatch detection and a resulting conflict which are likely localized in frontal brain areas. If the effect exists, it would indicate that our cognitive system tries to integrate the two subliminal facial expressions, and moreover that unconscious processes reach higher processing levels and interact with each other. Although sequential presentation of stimuli is often used in studies about the 
influence between conscious and unconscious processing, the concurrent presentation of subliminally emotional faces could be regarded as processing one face under the influence of the other face just as invisible stimuli could be modulated by concurrent management of attentional load [25]. This is an exploratory research, therefore, both ERP and fMRI techniques are used to see whether the assumed effect is stable.

Two experimental conditions were designed: 1) two simultaneously and subliminally presented faces of different unconscious valence (fearful versus happy face, DUV) and 2) two simultaneously and subliminally presented faces with the same unconscious valence (fearful or happy faces, SUV). DUV probably affects the N2 ERP component more differently than the SUV condition, since this component is sensitive to both subliminal face processing $[18,20]$ and conscious mismatch detection [26]. This N2 effect might be the result of activation differences in the frontal gyrus, because this structure is linked to conscious mismatch processing and unconscious incongruent priming [16,17,27,28].

\section{EXPERIMENT 1: ERP STUDY}

\subsection{Method}

\subsubsection{Participants}

Twenty five participants from Southwest University in China volunteered for this EEG study (12 women, 13 men; aged 18 - 25 years [mean age 22.6 years]). All participants were paid, gave written informed consent, were right-handed, had no history of current or past neurological or psychiatric illness, and had normal or corrected-to-normal vision.

\subsubsection{Stimuli}

A sample consisting of 40 images of happy facial expression (20 females, 20 males) and 40 images of fearful facial expression (20 females, 20 males) was collected from the Chinese Facial Affective Picture System [29]. The mean valence for fearful and happy faces was 2.73 (SD, 0.44) and 6.28 (SD, 0.61), respectively. The mean arousal for fearful and happy faces was 6.31 (SD, 1.22) and 5.46 (SD, 1.17), respectively. The scale range for valence and arousal was $1-9$. During the experiment, the face images were displayed centrally on a uniform grey background $(\mathrm{RGB}=192,192,192)$ and each face subtended approximately 4.3 (height) $\times 3.8$ (width) degrees of visual angle.

\subsubsection{Procedure}

The stimulus presentation sequence of an example trial is displayed in Figure 1. In the experiment, participants were asked to monitor a number and indicate if it was

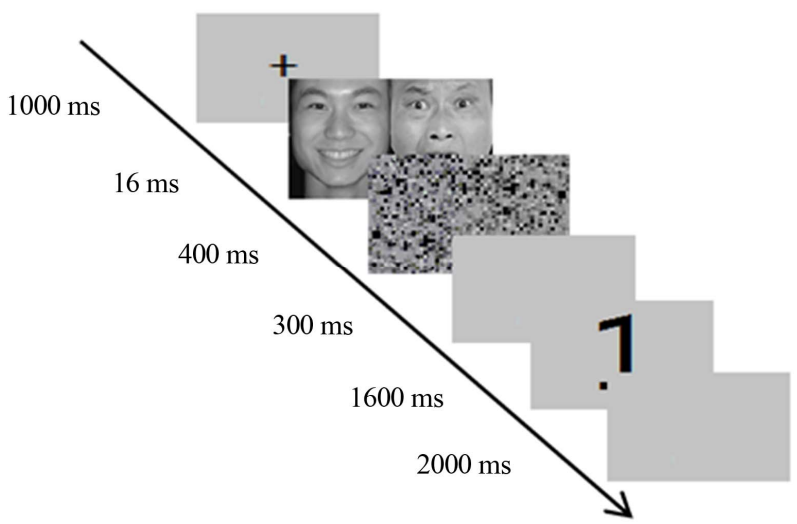

Figure 1. Schematic illustration of the ERP task. Participants were required to identify the numbers as odd or even, having been told that the stimuli prior to the numbers were distractions that they should ignore.

odd or even. First, a fixation cross appeared in the center of the screen for $1000 \mathrm{~ms}$. Subsequently two paired face images were presented side-by-side on the screen for 16 ms (one screen refresh), followed by a backward mask of two scrambled faces for $400 \mathrm{~ms}$. Following a blank screen of $300 \mathrm{~ms}$, a number was displayed for $1500 \mathrm{~ms}$. Participants were asked to indicate if the number was odd or even by pressing 1 or 2 with their right index and middle fingers respectively, as quickly and accurately as possible. The stimuli prior to the number were distractors that should be ignored. The responses were counterbalanced across subjects. At last, a blank screen appeared for $2000 \mathrm{~ms}$. During the experiment, the participants were seated in a quiet room facing a screen placed approximately in $100 \mathrm{~cm}$ distance from the eyes and were asked to sit still and blink as little as possible.

There were two conditions. The two faces had either different unconscious valences (DUV) or the same unconscious valence (SUV). To avoid any difference in low-level features, stimuli between DUV and SUV were matched (Figure 2). Thus, unconscious effects between these two conditions cannot be attributed to physical stimulus differences. Face pictures in a trial were of the same gender, and the ratio of odd to even numbers was one to one within each condition. First, the subjects were trained in five practice trials. Subsequently, two experimental blocks were run. Each one consisted of 80 trials, resulting in 80 trials per condition (DUV and SUV). The different conditions in each block were displayed randomly. Between the blocks, the subjects could take an appropriate rest.

After the ERP experiment, the participants were asked to report whether they saw something apart from the numbers, and then a forced-choice discrimination task was performed in order to test the participants' ability to recognize the masked faces. After a fixation cross, displayed for $1000 \mathrm{~ms}$, a face image was presented for 16 $\mathrm{ms}$, followed by a backward mask of a scrambled face 

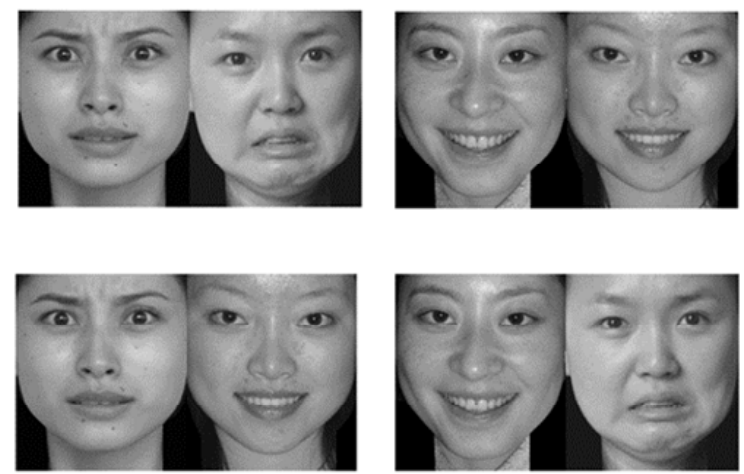

Figure 2. Examples of the pairing stimuli. Top: SUV condition. Bottom: DUV condition. The four faces for each condition were same, except for their match.

for $400 \mathrm{~ms}$. Then a pair of faces was presented. Participants were asked to determine which one was presented before. Before performing this task, participants were informed that the probability of the two faces being the test face was equal, and that only the accuracy not the speed of the response was important.

\subsubsection{ERP Recording and Analysis}

Brain electrical activity was recorded from $64 \mathrm{Ag} / \mathrm{AgCl}$ electrodes mounted in an elastic cap (Brain Products), electrodes at the left and right mastoids served as reference. The electrooculogram (EOG) was recorded from electrodes placed above and below the right eye (vertical), and at the right side of the right eye and the left side of the left eye (horizontal). All interelectrode impedances were kept below $5 \mathrm{k} \Omega$. The EEG and EOG were amplified using a $0.05-80 \mathrm{~Hz}$ bandpass and continuously sampled at $500 \mathrm{~Hz} /$ channel for offline analysis. Oculor artifacts (blinks and eye movements) were rejected offline. Trials with EOG artifacts (mean EOG voltage exceeding $\pm 80 \mu \mathrm{V}$ ) and those contaminated with artifacts due to amplifier clipping, bursts of electromyographic activity, or peak-to-peak deflection exceeding $\pm 80 \mu \mathrm{V}$ were excluded from averaging.

The EEG of the main experiment was epochized from -100 to $450 \mathrm{~ms}$ relative to the onset of the face stimulus. The first $100 \mathrm{~ms}$ served as baseline. The targeted N2 ERP component peaked around 220 to $230 \mathrm{~ms}$ with a maximum at left frontal-central electrodes (F1, F3, FC1, FC3, FC5 , C1, C3, C5). In order to define statistical differences at this component between the SUV and DUV condition mean amplitude values averaged in this timewindow across these electrodes were submitted to a paired t-test.

\subsection{Results}

\subsubsection{Visibility Test}

The participants reported not seeing the masked faces. In the measure of stimulus visibility, 23 of 25 participants scored at chance level (binomial test, $\mathrm{p}>0.05$ ), suggesting that these individuals were unable to perceive masked faces. Also at the group level, discrimination performance did not deviate from chance level (mean percentage correct $=48.96 \%, \mathrm{SD}=0.08, \mathrm{t}(22)=-0.61, \mathrm{p}$ $=0.55)$. The two participants that scored above chance level were excluded from further behavioral and ERP analysis.

\subsubsection{Number Judgment}

The reaction times (RTs) to the numbers for SUV and DUV were $596.6 \pm 146.1$ and $603.1 \pm 154.0 \mathrm{~ms}$, respectively. The effect of RTs was not significant, $\mathrm{t}(22)=1.42$, $\mathrm{p}=0.17$. In addition, the degrees of accuracy for SUV and DUV were $98.6 \% \pm 1.8 \%$ and $98.3 \% \pm 2.3 \%$, respectively. The effect was also insignificant, with $\mathrm{t}(22)=$ $-0.79, \mathrm{p}=0.43$.

\subsubsection{Electrophysiological Scalp Data}

The N2 component was significantly influenced by the stimulus type, $\mathrm{t}(22)=-2.27, p<0.05$, as the N2 amplitude for DUV trials was less negative than for SUV trials (Figure 3), indicating processing differences between identical and different subliminally perceived facial expressions.

\section{EXPERIMENT 2: fMRI STUDY}

\subsection{Method}

\subsubsection{Participants}

As paid volunteers, eighteen new adults that did not contribute data to the ERP study (7 women, 11 men) aged 19 - 28 years (mean age, 23.6 years) from Southwest University in China participated in this experiment. All participants gave written informed consent, were righthanded, had no history of current or past neurological or psychiatric illness, and had normal or corrected-to-normal vision.

\subsubsection{Stimuli}

Same as those used in ERP study.
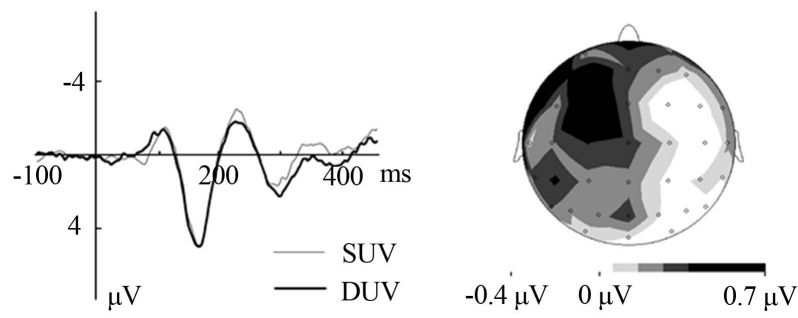

Figure 3. ERP averaged across eight electrodes (F1, F3, FC1, FC3, FC5, C1, C3, C5), neativity is plotted upwards. The N2 component peaked around 220 to $230 \mathrm{~ms}$. Topographical voltage map of the N2 effect (DUV-SUV) in the averaged time window from 220 to $230 \mathrm{~ms}$ with the left-fronto-central maximum. 


\subsubsection{Procedure}

The experimental task, stimulus delivery, and recording of behavioral responses were carried out with E-prime 2.0 Software (Psychology Software Tools, Inc.

http://www.pstnet.com). The face images were displayed centrally on a uniform grey background $(\mathrm{RGB}=192$, $192,192)$ and each face subtended a visual angle of approximately 4.3 (height) $\times 3.8$ (width) degrees. Stimuli were displayed on a back-projection screen placed at the back of the scanner bore, which was viewed by the participants via a mirror attached to the head-coil. Manual responses were recorded using an MRI-compatible button box.

In the experiment, participants were required to monitor a number for vigilance maintenance and indicate whether the number was odd or even by pressing 1 or 2 , respectively, in the button box. The stimulus-responseassignment was counterbalanced across participants. The trial sequence was as follows (Figure 4): a forward mask of two scrambled faces was first displayed for $384 \mathrm{~ms}$, and then two paired face images were presented side-byside on the screen for $16 \mathrm{~ms}$ (one screen refresh), followed by a backward mask for $384 \mathrm{~ms}$. For a better BOLD signal, paired face images of the same conditions and the following masks were repeated five times. The last mask was displayed for $400 \mathrm{~ms}$ in a trial, followed by the target number for $1600 \mathrm{~ms}$. Note that no image was displayed twice in a trial. Between trials, a central fixation cross was displayed for a jittered inter-trial interval of 2 $6 \mathrm{~s}$.

The two paired face images were presented in the DUV and the SUV condition. Stimuli between DUV and SUV were matched as described in the ERP study (Figure 2). In a single trial of each condition, only one type

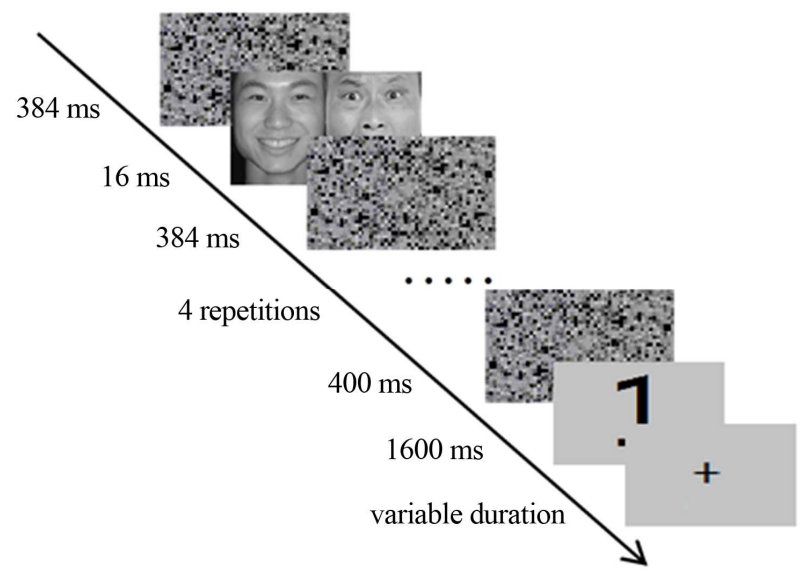

Figure 4. Schematic illustration of the fMRI task. For better blood oxygenation level-dependent (BOLD) signal, the paired face images of one type (DUV and SUV) were successively repeated five times. However, there was no face image displayed twice in a single trial. of paired face images was displayed and no face image was displayed twice. In the DUV condition, the happy and fearful faces interchanged their positions successively between five subliminal displays to maximize the mismatch which had two sources: one from the concurrent mismatch, another from the successive mismatch. In the SUV condition, happy or fearful face pairs were displayed five times. In addition, the face images in a trial were of the same gender, and the ratio of odd to even numbers was one to one within each condition. The participants were asked to make the odd/even judgment as quickly and accurately as possible. The participants were also informed that the stimuli prior to the number should be ignored. There were two runs, each contained 20 trials of DUV and 20 trials of SUV (10 trials of happy or fearful face, respectively). The different conditions in each run were displayed randomly.

In order to test the participants' ability to see the masked faces, the participants were asked after fMRI scanning whether or not they saw something apart from the numbers, and then a forced-choice discrimination task was performed while still lying in the scanner. Stimuli and trial times were similar to those used in the formal fMRI experiment. However, one face instead of the paired faces was presented, and the same face was repeated five times in a trial. After the presentation of the last mask, a pair of faces, of which one was the same face presented before, was presented left and right of the fixation. Participants were asked to determine which one was presented previously. The two faces remained on the screen until the participants made a response. There were 60 trials in the discrimination task. Before performing this task, participants were informed that the probability of the two faces being the test face was equal, and that only the accuracy not the speed of the response was important.

\subsection{4. fMRI Data Acquisition}

Imaging data were acquired with a 3-T Siemens Trio Scanner (Siemens Medical Systems, Erlangen, Germany) using a 12-channel birdcage headcoil. Two functional scans were acquired using an echo planar imaging (EPI) sequence $\left(\mathrm{TR}=2000 \mathrm{~ms}, \mathrm{TE}=30 \mathrm{~ms}\right.$, flip angle: $90^{\circ}$; field of view: $220 \times 220 \mathrm{~mm}^{2}$; matrix size: $64 \times 64$ ). Each functional volume consisted of 32 axial slices of 3 $\mathrm{mm}$ thickness with $1 \mathrm{~mm}$ gap between slices. Each scan lasted 6 min and consisted of 180 volumes. Two dummy scans were performed prior to the image acquisition to eliminate signals arising from progressive saturation.

\subsection{5. fMRI Data Analysis}

All pre-processing and statistical analyses were carried out using SPM8

(http://www.fil.ion.ucl.ac.uk/spm/software/spm8/). Prior to pre-processing, the first three volumes of each run 
were discarded. For each participant, functional images were spatially aligned and slice-time corrected to the first volume of the first run, and then normalized to the Montreal Neurological Institute (MNI) template brain. The normalized functional images (resampled at $3 \mathrm{~mm}^{3}$ ) were spatially smoothed with a Gaussian kernel of full widthhalf-maximum of $8 \mathrm{~mm}^{3}$. A $128 \mathrm{~s}$ temporal high pass filter was applied to remove low-frequency scanner artifacts. Using a first-order autoregressive model (AR-1), temporal autocorrelations were estimated using restricted maximum likelihood estimates of variance components, and maximum likelihood estimates of the activations were formed using the previously resulting non-sphericity [30].

For statistical analysis, we constructed models of two separate regressors coding for onsets and durations ( $2 \mathrm{~s})$ of DUV and SUV. The regressors were convolved with SPM8's canonical hemodynamic response function (HRF) and the models were regressed against the observed BOLD data. For each participant, we extracted mean activation estimates (\% signal change) for DUV and SUV trials from their individual MFG which activated in whole-brain analysis, using Marsbar software

(http://marsbar.sourceforge.net/), and presented the mean activation estimates across all subjects (Figure 5).

All 18 participants were included in these analyses except for one run of one participant because the behavioral results were overwritten. The fMRI analysis included (DUV-SUV) and (SUV-DUV). Both comparesons were thresholded at $\mathrm{p}<0.05$ (small volume corrected and cluster size $>5$ voxels). In addition, the contrasts of (SUV-DUV) was also evaluated at a more liberal threshold $(\mathrm{p}<0.01$ uncorrected and cluster size $>0$ voxels) to see to what extent there would be no activation.

\subsection{Results}

\subsubsection{Visibility Test}

The participants reported not seeing the masked faces. In the measure of stimulus visibility, all 18 participants
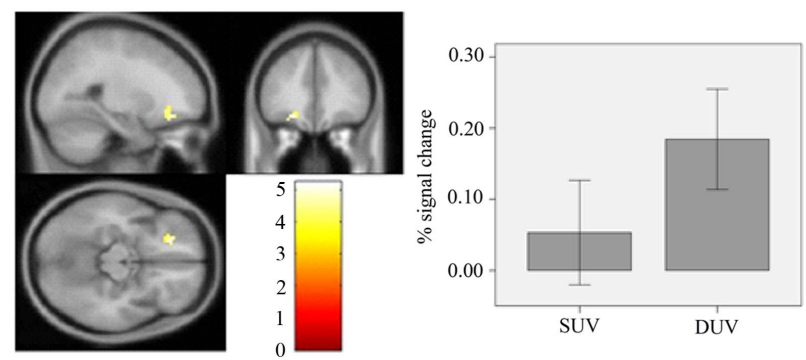

Figure 5. Left: Comparison of the results of the DUV with SUV. The image shows activation in the left middle frontal gyrus $(x=-21, y=35$, and $z=-14)$ at a threshold of $\mathrm{p}<0.05$ (small volume corrected, cluster size $>5$ voxels). Right: The mean and SE of \% signal change for SUV and DUV. scored at chance level (binomial test, $p>0.05$ ), suggesting that these individuals were unable to perceive the masked faces. Also at the group level, discrimination performance did not deviate from chance level (mean percentage correct $=51.00 \%, \mathrm{SD}=0.06$, $\mathrm{t}(17)=0.74, \mathrm{p}=0.47)$.

\subsubsection{Number Judgment}

The reaction times (RTs) to the numbers for SUV and DUV were $787.2 \pm 144.9$ and $787.6 \pm 135.5 \mathrm{~ms}$, respectively. The effect of facial valence (same vs. different) was not significant, $\mathrm{t}(17)=-0.06, \mathrm{p}=0.95$. In addition, the accuracy rates for SUV and DUV were $96.4 \% \pm$ $7.2 \%$ and $96.3 \% \pm 7.0 \%$, respectively. The difference was also insignificant, with $\mathrm{t}(17)=0.12, \mathrm{p}=0.91$.

\subsection{3. fMRI Results}

Comparing the DUV with SUV trials, the fMRI results with whole-brain analysis only showed activation in the left middle frontal gyrus (MFG) $(\mathrm{x}=-21, \mathrm{y}=35$, and $\mathrm{z}$ $=-14 ; 21$ voxels, and $\mathrm{t}=5.26)$ at a threshold of $\mathrm{p}<0.05$ (small volume corrected, cluster size $>5$ voxels) (Figure 5). The result was further visualized using xjView toolbox (http://www.alivelearn.net/xjview). Thus, the hypothesis of interactions between unconscious processes was supported. Furthermore, comparing SUV with DUV, no activation was observed even at an uncorrected threshold of $\mathrm{p}<0.01$ (cluster size $>0$ voxels).

\section{DISCUSSION}

In this study, we investigated whether concurrent unconscious processes interact. Both ERP and fMRI data indicated that subliminally presented faces of different valence are differently processed than faces of the same valence. In the first ERP experiment, DUV elicited a smaller N2 as compared to SUV. In the second fMRI experiment, the left MFG was activated under DUV condition in comparison to SUV condition. We assume that in comparison to two subliminal facial expressions of the same valence, two subliminal facial expressions of different valence are subconsciously perceived as a mismatch and elicit a conflict. Previous studies demonstrated that supraliminally presented bilateral face stimuli lead to interhemispheric cooperation $[31,32]$. Therefore the effect in our studies would indicate that at a certain processing level the brain tries to integrate the expression analyses of the two faces, which likely supports the idea of interactions between unconscious processes. In the following, the ERP and fMRI results as well as theoretical implications are discussed.

\subsection{Discussion about ERP and fMRI Results}

Because stimuli between DUV and SUV were matched 
in both ERP and fMRI studies, the results cannot be attributed to differences in low-level features. Instead, we assume that the results reflect unconscious detection of a mismatch. In the ERP study, the N2 component correlated with unconscious mismatch processing and had a left anterior maximum. The N2 was smaller under DUV than under SUV conditions. In contrast to the posterior $\mathrm{N} 2$, which is related to the degree of attention required for processing stimuli in the visual modality [33], the anterior N2 was considered to be associated with mismatch or cognitive control [26,34]. For example, the N2 was enhanced for incongruent flankers in Flanker tasks [35] and for no-go trials in go/no-go tasks [34,36]. In comparison with strong evidence that the $\mathrm{N} 2$ associated with cognitive control is located in anterior cingulate area $[37,38]$, there are studies that did not find a novelty/mismatch effect in the anterior cingulate area but in the frontal cortex using visual oddball paradigms, which are known to elicit robust N2 components $[39,40]$. Considering the activation in MFG in the fMRI experiment, it is reasonable to assume that the N2 in the ERP study reflects unconscious mismatch detection rather than cognitive control. Interestingly, in our study the N2 was small-ler under the DUV than under the SUV condition which is at odds with studies investigating conscious stimulus processing. Thus, one possible assumption could be that ERP effects to subthreshold processing differ from ERP effects to supraliminal perception. This is quite speculative, since there is to the best of our knowledge no study that actually measured ERPs to unconsciously perceived primes but only to conscious targets. Comparing two identical studies by Kiefer and colleagues, in which unconscious semantic priming was investigated [41], ERP effects in the N400 component were reversed, when unconscious prime processing was modulated by top-down influences [42]. Future studies should aim at investigating this issue explicitly.

In the fMRI experiment, the left MFG was stronger activated under the DUV condition in comparison to the SUV condition. The MFG has been associated with mismatch processing across a wide range of experimental paradigms. For example, MFG activation was related to auditory mismatch processing when comparing blocks that contained rare duration-deviant tones with blocks of a series of standard tones [27]. In a study with a coherent object in a scenic context and an incoherent object in a scenic context, bilateral MFG was activated during the incoherent condition [28]. Moreover, transcranial magnetic stimulation of the left frontal cortex significantly reduced neuronal and behavioral repetition priming effects (which resembles our SUV condition) [43]. In addition, mid-dorsolateral prefrontal cortex was activated during unconscious priming when comparing the incongruent priming condition with the congruent priming condition [16]. This prefrontal activation was assumed to be related to increasing cognitive demands or to a high level of cognitive conflict [16]. Furthermore, it was found that correlations between neuronal and behavioral priming were specific for faces in the left middle frontal gyrus [44]. Therefore, we assume that the left MFG is involved in unconscious mismatch processing, maybe trying to integrate the two conflicting subliminal facial expressions.

In the DUV condition of the fMRI experiment, the happy and fearful faces interchanged their positions successively between five subliminal displays to maximize the mismatch. Therefore, the mismatch process in DUV condition has two sources: one from the concurrent mismatch, another from the successive mismatch. The additional successive mismatch in fMRI experiment compared to ERP experiment could be related to the reversed result pattern between the two studies. This is a caveat that needs further corroboration.

To summarize, the ERP and fMRI results showed that perceiving two facial expressions of different valence subliminally elicited different unconscious processes as compared to perceiving two facial expressions of the same valence, which might reflect the interaction of unconscious processes due to unconscious mismatch detection.

\subsection{Theoretical Implications}

The existence of interactions between unconscious processes could amend the theory of global neuronal workspace. In a study about the access threshold to consciousness, Del Cul and colleagues proposed that 1) masked and unmasked stimuli initially elicit identical visual activations, and 2) that the amount of masking is negatively correlated to the depth of processing (i.e. unconscious processes are restricted to occipital and parietal brain areas) [15]. However, there is an exceptional study that showed that subliminal words activated the anterior cortex relative to subliminal pseudowords [45]. This study is in line with our present findings, which demonstrate that unconscious processing is not limited to the posterior brain area.

In addition, the theory of global neuronal workspace proposes two computational spaces: 1) the global workspace, which is composed of distributed and interconnected neurons with long-range axons, and 2) specialized and modular perceptual, motor, memory, evaluative, and attentional processors [46]. Dehaene et al. suggested that automatic processing should activate specialized processors without requiring coordination by global workspace neurons [46]. However, some works indicate that unconscious processes are not necessarily automatic but influenced by top-down factors, like task sets for instance $[42,47,48]$. Accordingly, one could assume that global workspace neurons are also active during some uncon- 
scious processes. This would allow not only for sequential activation of modular processors but also for more complex unconscious cognitive processes like for example the demonstrated unconscious mismatch detection. Just thinking of the existence of interactions between the unconscious perceptual, motor, memory, evaluative, and attentional processors, this could lead to abundant interactions between them, and many phenomena could be well explained. For instance, it could be that persons with obsessive-compulsive disorders cannot consciously control the unconscious memory/evaluative influences on unconscious motor processes. Such an interpretation is corroborated by work demonstrating that motor preparatory processes occur before we have the conscious will to act [49].

\section{CONCLUSION}

To conclude, this study extends previous results on relationship between conscious and unconscious cognitive processing to unconscious interactions between unconscious cognitive processes. However, we are just at the beginning to understand how different unconscious processes influence each other. Future research needs to further verify and specify its phenomenal and theoretical use.

\section{ACKNOWLEDGEMENTS}

This research was supported by the National Natural Science Foundation of China (31170983), the Fundamental Research Funds for the Central Universities (NSKD11002) and the Fundamental Research Funds of China West Normal University.

\section{REFERENCES}

[1] Custers, R. and Aarts, H. (2010) The unconscious will: How the pursuit of goals operates outside of conscious awareness. Science, 329, 47-50.

http://dx.doi.org/10.1126/science.1188595

[2] de Gardelle, V., Charles, L. and Kouider, S. (2011) Perceptual awareness and categorical representation of faces: Evidence from masked priming. Consciousness and Cognition, 20, 1272-1281.

http://dx.doi.org/10.1016/j.concog.2011.02.001

[3] Linser, K. and Goschke, T. (2007) Unconscious modulation of the conscious experience of voluntary control. Cognition, 104, 459-475.

http://dx.doi.org/10.1016/j.cognition.2006.07.009

[4] Luo, Q., Peng, D.L., Jin, Z., Xu, D., Xiao, L.H. and Ding, G.S. (2004) Emotional valence of words modulates the subliminal repetition priming effect in the left fusiform gyrus: An event-related fMRI study. Neuroimage, 21, 414-421.

http://dx.doi.org/10.1016/j.neuroimage.2003.09.048

[5] Yamadaa, M. and Jean, D.B. (2009) Unconscious affective processing and empathy: An investigation of sub- liminal priming on the detection of painful facial expressions. Pain, 143, 71-75.

http://dx.doi.org/10.1016/i.pain.2009.01.028

[6] Jolij, J. and Lamme, V.A.F. (2005) Repression of unconscious information by conscious processing: Evidence from affective blindsight induced by transcranial magnetic stimulation. Proceedings of the National Academy of Sciences of the United States of America, 102, 1074710751. http://dx.doi.org/10.1073/pnas.0500834102

[7] Kanai, R., Tsuchiya, N. and Verstraten, F.A.J. (2006) The scope and limits of top-down attention in unconscious visual processing. Current Biology, 16, 2332-2336. http://dx.doi.org/10.1016/j.cub.2006.10.001

[8] Rauss, K.S., Pourtois, G., Vuilleumier, P. and Schwartz, S. (2009) Attentional load modifies early activity in human primary visual cortex. Human Brain Mapping, 30, 1723-1733. http://dx.doi.org/10.1002/hbm.20636

[9] Schmidt, F. and Schmidt, T. (2010) Feature-based attention to unconscious shapes and colors. Attention, Perception, \& Psychophysics, 72, 1480-1494. http://dx.doi.org/10.3758/APP.72.6.1480

[10] Shin, K., Stolte, M. and Chong, S.C. (2009) The effect of spatial attention on invisible stimuli. Attention, Perception, \& Psychophysics, 71, 1507-1513. http://dx.doi.org/10.3758/APP.71.7.1507

[11] Watanabe, M., Cheng, K., Murayama, Y., Ueno, K., Asamizuya, T. and Tanaka, K., et al. (2011) Attention but not awareness modulates the BOLD signal in the human V1 during binocular suppression. Science, 334, 829-831. http://dx.doi.org/10.1126/science.1203161

[12] Jiang, Y., Costello, P., Fang, F., Huang, M. and He, S. (2006) A gender- and sexual orientation-dependent spatial attentional effect of invisible images. Proceedings of the National Academy of Sciences of the United States of America, 103, 17048-17052. http://dx.doi.org/10.1073/pnas.0605678103

[13] Lin, J.Y., Murray, S.O. and Boynton, G.M. (2009) Capture of attention to threatening stimuli without perceptual awareness. Current Biology, 19, 1118-1122. http://dx.doi.org/10.1016/j.cub.2009.05.021

[14] Dehaene, S., Changeux, J., Naccache, L., Sackur, J. and Sergent, C. (2006) Conscious, preconscious, and subliminal processing: A testable taxonomy. Trends in Cognitive Sciences, 10, 204-211. http://dx.doi.org/10.1016/j.tics.2006.03.007

[15] Del Cul, A., Baillet, S. and Dehaene, S. (2007) Brain dynamics underlying the nonlinear threshold for access to consciousness. PLOS Biology, 5, e260. http://dx.doi.org/10.1371/journal.pbio.0050260

[16] Lau, H.C. and Passingham, R.E. (2007) Unconscious activation of the cognitive control system in the human prefrontal cortex. The Journal of Neuroscience, 27, 58055811. http://dx.doi.org/10.1523/JNEUROSCI.4335-06.2007

[17] van Gaal, S., Ridderinkhof, K.R., Scholte, H.S. and Lamme, V.A.F. (2010) Unconscious activation of the prefrontal no-go network. The Journal of Neuroscience, 30, 4143-4150.

http://dx.doi.org/10.1523/JNEUROSCI.2992-09.2010 
[18] Balconi, M. and Lucchiari, C. (2005) In the face of emotions: Event-related potentials in supraliminal and subliminal facial expression recognition. Genetic, Social, and General Psychology Monographs, 131, 41-69. http://dx.doi.org/10.3200/MONO.131.1.41-69

[19] Dimberg, U., Thunberg, M. and Elmehed, K. (2000) Unconscious facial reactions to emotional facial expressions. Psychological Science, 11, 86-89. http://dx.doi.org/10.1111/1467-9280.00221

[20] Kiss, M. and Eimer, M. (2008) ERPs reveal subliminal processing of fearful faces. Psychophysiology, 45, 318326. http://dx.doi.org/10.1111/j.1469-8986.2007.00634.x

[21] Liddell, B.J., Williams, L.M., Rathjen, J., Shevrin, H. and Gordon, E. (2004) A temporal dissociation of subliminal versus supraliminal fear perception: An event-related potential study. Journal of Cognitive Neuroscience, 16, 479486. http://dx.doi.org/10.1162/089892904322926809

[22] Pessoa, L. (2005) To what extent are emotional visual stimuli processed without attention and awareness? Current Opinion in Neurobiology, 15, 188-196. http://dx.doi.org/10.1016/j.conb.2005.03.002

[23] Tamietto, M. and de Gelder, B. (2010) Neural bases of the non-conscious perception of emotional signals. $\mathrm{Na}$ ture Reviews Neuroscience, 11, 697-709. http://dx.doi.org/10.1038/nrn2889

[24] Whalen, P.J., Rauch, S.L., Etcoff, N.L., McInerney, S.C., Lee, M.B. and Jenike, M.A. (1998) Masked presentations of emotional facial expressions modulate amygdala activity without explicit knowledge. The Journal of Neuroscience, 18, 411-418.

[25] Bahrami, B., Lavie, N. and Rees, G. (2007) Attentional load modulates responses of human primary visual cortex to invisible stimuli. Current Biology, 17, 509-513. http://dx.doi.org/10.1016/j.cub.2007.01.070

[26] Folstein, J.R. and Van Petten, C. (2008) Influence of cognitive control and mismatch on the N2 component of the ERP: A review. Psychophysiology, 45, 152-170. http://dx.doi.org/10.1111/j.1469-8986.2007.00602.x

[27] Schall, U., Johnston, P., Todd, J., Ward, P.B. and Michie, P.T. (2003) Functional neuroanatomy of auditory mismatch processing: An event-related fMRI study of duration-deviant oddballs. Neuroimage, 20, 729-736. http://dx.doi.org/10.1016/S1053-8119(03)00398-7

[28] Martens, U., Trujillo-Barreto, N. and Gruber, T. (2011) Perceiving the tree in the woods: Segregating brain responses to stimuli constituting natural scenes. The Journal of Neuroscience, 31, 17713-17718. http://dx.doi.org/10.1523/JNEUROSCI.4743-11.2011

[29] Luo, Y.J., Huang, Y.X., Li, X.Y. and Li, X.B. (2006) Effects of emotion on cognitive processing: Series of event-related potentials study. Advances in Psychological Science (Chinese), 14, 505-510.

[30] Egner, T. (2011) Right ventrolateral prefrontal cortex mediates individual differences in conflict-driven cognitive control. Journal of Cognitive Neuroscience, 23, 39033913. http://dx.doi.org/10.1162/jocn_a_00064

[31] Mohr, B., Landgrebe, A. and Schweinberger, S.R. (2002) Interhemispheric cooperation for familiar but not unfa- miliar face processing. Neuropsychologia, 40, 1841-1848. http://dx.doi.org/10.1016/S0028-3932(02)00040-4

[32] Tamietto, M., Corazzini, L.L., de Gelder, B. and Geminiani, G. (2006) Functional asymmetry and interhemispheric cooperation in the perception of emotions from facial expressions. Experimental Brain Research, 171, 389-404. http://dx.doi.org/10.1007/s00221-005-0279-4

[33] Suwazono, S., Machado, L. and Knight, R.T. (2000) Predictive value of novel stimuli modifies visual event-related potentials and behavior. Clinical Neurophysiology, 111, 29-39. http://dx.doi.org/10.1016/S1388-2457(99)00186-8

[34] van Gaal, S., Lamme, V.A.F., Fahrenfort, J.J. and Ridderinkhof, K.R. (2011) Dissociable brain mechanisms underlying the conscious and unconscious control of behavior. Journal of Cognitive Neuroscience, 23, 91-105. http://dx.doi.org/10.1162/jocn.2010.21431

[35] Heil, M., Osman, A., Wiegelmann, J., Rolke, B. and Hennighausen, E. (2000) N200 in the Eriksen-task: Inhibitory executive processes? Journal of Psychophysiology, 14, 218-225. http://dx.doi.org/10.1027//0269-8803.14.4.218

[36] Donkers, F.C.L. and van Boxtel, G.J.M. (2004) The N2 in go/no-go tasks reflects conflict monitoring not response inhibition. Brain and Cognition, 56, 165-176. http://dx.doi.org/10.1016/j.bandc.2004.04.005

[37] Debener, S., Ullsperger, M., Siegel, M., Fiehler, K., Von Cramon, D.Y. and Engel, A.K. (2005) Trial-by-trial coupling of concurrent electroencephalogram and functional magnetic resonance imaging identifies the dynamics of performance monitoring. The Journal of Neuroscience, 25, $11730-11737$. http://dx.doi.org/10.1523/JNEUROSCI.3286-05.2005

[38] Holroyd, C.B. and Coles, M.G.H. (2002) The neural basis of human error processing: Reinforcement learning, dopamine, and the error-related negativity. Psychological Review, 109, 679-709. http://dx.doi.org/10.1037/0033-295X.109.4.679

[39] Kiehl, K.A., Laurens, K.R., Duty, T.L., Forster, B.B. and Liddle, P.F. (2001) An event-related fMRI study of visual and auditory oddball tasks. Journal of Psychophysiology, 15, 221-240.

[40] Kirino, E., Belger, A., Goldman-Rakic, P. and McCarthy, G. (2000) Prefrontal activation evoked by infrequent target and novel stimuli in a visual target detection task: An event-related functional magnetic resonance imaging study. The Journal of Neuroscience, 20, 6612-6618.

[41] Kiefer, M. (2002) The N400 is modulated by unconsciously perceived masked words: Further evidence for an automatic spreading activation account of N400 priming effects. Cognitive Brain Research, 13, 27-39. http://dx.doi.org/10.1016/S0926-6410(01)00085-4

[42] Kiefer, M. and Martens, U. (2010) Attentional sensitization of unconscious cognition: Task sets modulate subsequent masked semantic priming. Journal of Experimental Psychology: General, 139, 464-489. http://dx.doi.org/10.1037/a0019561

[43] Wig, G.S., Grafton, S.T., Demos, K.E. and Kelley, W.M. (2005) Reductions in neural activity underlie behavioral components of repetition priming. Nature Neuroscience, 
8, 1228-1233.

[44] Schacter, D.L., Wig, G.S. and Stevens, W.D. (2007) Reductions in cortical activity during priming. Current Opinion in Neurobiology, 17, 171-176.

http://dx.doi.org/10.1016/j.conb.2007.02.001

[45] Diaz, M.T. and McCarthy, G. (2007) Unconscious word processing engages a distributed network of brain regions. Journal of Cognitive Neuroscience, 19, 1768-1775. http://dx.doi.org/10.1162/jocn.2007.19.11.1768

[46] Dehaene, S., Kerszberg, M. and Changeux, J.P. (1998) A neuronal model of a global workspace in effortful cognitive tasks. Proceedings of the National Academy of Sciences of the United States of America, 95, 14529-14534. http://dx.doi.org/10.1073/pnas.95.24.14529
[47] Martens, U., Ansorge, U. and Kiefer, M. (2011) Controlling the unconscious: Attentional task sets modulate subliminal semantic and visuomotor processes differentially. Psychological Science, 22, 282-291. http://dx.doi.org/10.1177/0956797610397056

[48] Tu, S., Qiu, J., Martens, U. and Zhang, Q.L. (2013) Category-Selective attention modulates unconscious processes in the middle occipital gyrus. Consciousness and Cognition, 22, 479-485.

http://dx.doi.org/10.1016/j.concog.2013.02.007

[49] Haggard, P. and Libet, B. (2001) Conscious intention and brain activity. Journal of Consciousness Studies, 8, 47 64. 